\title{
Metabolic Syndrome and Its Components in Psoriatic Arthritis
}

\author{
Roaa Aljohani \\ Department of Medicine, College of Medicine, Taibah University, Madinah, Saudi Arabia \\ Correspondence: Roaa Aljohani, Email roaahmad@taibahu.edu.sa
}

\begin{abstract}
Psoriatic arthritis (PsA) is a well-known inflammatory disorder with a wide variety of phenotypes that extend beyond the joints. It has been defined as an immune-mediated disorder in which Th-1 and Th-17 cells play a key role. It has been associated with an elevated risk of metabolic syndrome (MetS), which is characterized by abdominal obesity, hypertension, hyperglycemia, and hyperlipidemia. While the exact pathophysiology of the link between PsA and MetS has yet to be precisely determined, persistence of inflammatory abnormalities, with overexpression of pro-inflammatory cytokines, might be the cause. Studies have consistently emphasized the strong association between elevated risk of developing cardiovascular disease and MetS in individuals with underlying PsA. The literature has also shown an association between the increased PsA severity and the increased frequency of MetS components. This association has important clinical consequences when treating patients with PsA. Therefore, screening programs should be implemented for PsA patients to evaluate whether they have MetS, and appropriate treatment should be given to manage cardiometabolic risk factors. Patients should also be closely monitored for potential adverse treatment effects on co-morbidities. This article summarizes the evidence of associations between several components of MetS and PsA and analyzes the impact of treatment on these factors.
\end{abstract}

Keywords: psoriatic arthritis, metabolic syndrome, cardiovascular disease, treatment

\section{Introduction}

Psoriatic arthritis (PsA) is a chronic autoinflammatory condition distinguished by a variety of clinical phenotypes. It is most frequently associated with patients who have psoriasis (PsO). It is a devastating form of spondyloarthropathy that significantly affects patients' life and increases cardiovascular mortality and mortality in general. ${ }^{1,2}$ Based on a rising awareness and knowledge of the immunologic mechanism, psoriatic disease is increasingly being regarded as a systemic disease, the consequences of which impact more than skin and joint health. ${ }^{3,4}$ The interest in determining the cardiovascular (CV) disease risk factors linked to PsA and PsO has intensified, with most of the early data and the underlying pathogenetic theories informed by the literature on rheumatoid arthritis (RA). ${ }^{5,6}$ Retrospective and prospective data from the large observational cohort, and evidence gleaned from various imaging techniques, show that PsO and PsA are linked to increased CV risk. ${ }^{4,7,8}$ Previous studies also indicate that $\mathrm{CV}$ diseases are the leading etiology for deaths in PsA patients, accounting for between $20 \%$ and $56 \%$ of those deaths. ${ }^{9,10}$ A relationship has been established in previous studies between increased risk of contracting CV disease in PsA and the existence of cardiometabolic risk factors, namely high blood pressure, adiposity, diabetes, hyperlipidemia, and chronic system inflammation. ${ }^{11}$ Moreover, the prevalence of MetS, described as an amalgamation of the risk factors mentioned above, is also higher in individuals diagnosed with PsA.

Metabolic syndrome denotes a collection of metabolic risk factors that increase the probability that CV disease will develop. Since the 1940s, there has been evidence that some metabolic abnormalities are associated with CV disease. Insulin resistance (IR), hypertension (HTN), abnormal cholesterol levels, and obesity are examples of these metabolic factors. 
Metabolic syndrome is a grouping of five traditional CV risk factors. There are five distinct MetS definitions. ${ }^{12-14}$ However, it has been proven that the various definitions of MetS are comparable in terms of prognosis and therapy. ${ }^{15}$ According to the most widely used current guidelines, which were revised by the National Heart, Lung, and Blood Institute (NHLBI) as well as the American Heart Association (AHA) in the United States in 2005, the diagnosis of MetS can be made if an individual has a minimum of three out of five of the conditions listed in Table $1 .{ }^{13}$

\section{Metabolic Syndrome and Psoriatic Arthritis}

Metabolic syndrome is increasing in importance as a worldwide health concern. It is typically asymptomatic but significantly influences general health, mainly by increasing the risk of CV disease.

Psoriatic arthritis, Metabolic syndrome, and atherosclerosis may have overlapping inflammatory pathways and genetic predispositions in their underlying pathophysiology (Figure 1). There is an increased prevalence of chronic inflammation mediated by Th1 and Th-17 with cytokine dysregulation in PsA. ${ }^{16,17}$

The pathogenesis of PsA is complicated and poorly known; The most widely acknowledged hypothesis involves an interplay between genetic predisposition and environmental exposures, resulting in immune-inflammatory pathway dysfunction. In individuals with a certain genetic history, the gut microbiota may be the missing piece in pathogenesis of PsA.

Recent evidence has emerged supporting the gut microbiome's pathogenic role in a different immune-mediated inflammatory disorders, such as diabetes mellitus, spondyloarthritis, and CV disease, the majority of which are wellknown comorbidities of $\mathrm{PsO}$ and PsA. ${ }^{18-20}$

So far, several studies on the intestinal microbiome of psoriatic patients have already been published, and four of them have demonstrated an imbalance between the two most prevalent intestinal flora, Firmicutes $(\mathrm{F})$ and Bacteroidetes (B), with an increased F/B ratio. ${ }^{21}$ This kind of gut dysbiosis has been linked to diabetes, obesity, and CV disease. ${ }^{22-24}$ Moreover, the presence of intestinal firmicutes was linked to a higher BMI and greater levels of the proatherogenic chemical trimethylamine-N-oxide, that raises the risk of $\mathrm{CV}$ events by enhancing endothelial dysfunction and interfering with cholesterol homeostasis at several levels. ${ }^{25,26}$

Furthermore, considering the comparable pathogenic aspects of obesity and psoriatic disease, both of which are characterized by similar cytokine profiles, an elevated F/B ratio might be another significant factor contributing to the pathogenesis of both of these diseases. ${ }^{27}$

Insulin resistance can be caused by a release of different cytokines, for example, tumor necrosis factor- $\alpha$, interleukin17, or interleukin- $6{ }^{28}$ Leptin is another adipokine that has a role in MetS and PsA pathogenesis. ${ }^{29}$ Increased BMI and IR have been linked to a high level of leptin. ${ }^{30}$ Endothelial dysfunction and the development of atherosclerotic plaques are caused by these inflammatory cytokines, which produce adhesion molecules and an elevated risk of CVS events in PsA. $^{31}$

Table I Definition of Metabolic Syndrome According to AHA/NHLBI Revised in 2005

\begin{tabular}{|l|l|}
\hline Criteria & Definition (Any 3 of 5 Criteria as Listed Below) \\
\hline $\begin{array}{l}\text { Central obesity } \\
\begin{array}{l}\text { Male } \\
\text { Female }\end{array}\end{array}$ & $\begin{array}{l}\text { WC }>40 \text { inches }(\geq 102 \mathrm{~cm}) \\
\text { WC }>35 \text { inches }(\geq 88 \mathrm{~cm}) .\end{array}$ \\
\hline Hyperglycemia & Fasting glucose $\geq 100 \mathrm{mg} / \mathrm{dl}(6.1 \mathrm{mmol} / \mathrm{L})$, or on treatment \\
\hline $\begin{array}{l}\text { HDL-cholesterol } \\
\text { Male }\end{array}$ & $\mathrm{HDL}-\mathrm{C}<40 \mathrm{mg} / \mathrm{dl}(1.033 \mathrm{mmol} / \mathrm{L})$ \\
Female & $\mathrm{HDL}-\mathrm{C}<50 \mathrm{mg} / \mathrm{dl}(1.29 \mathrm{mmol} / \mathrm{L})$ or on treatment \\
\hline $\begin{array}{l}\text { Hypertriglyceridemia } \\
\text { Hypertension }\end{array}$ & $\begin{array}{l}\mathrm{TG} \geq 150 \mathrm{mg} / \mathrm{dl}(1.7 \mathrm{mmol} / \mathrm{L}) \text { or being on therapy } \\
\mathrm{BP} \geq 130 / 85, \text { or on treatment }\end{array}$ \\
\hline
\end{tabular}

Abbreviations: WC, waist circumstances; HDL-C, high density lipoprotein; TG, triglyceride; BP, blood pressure. 


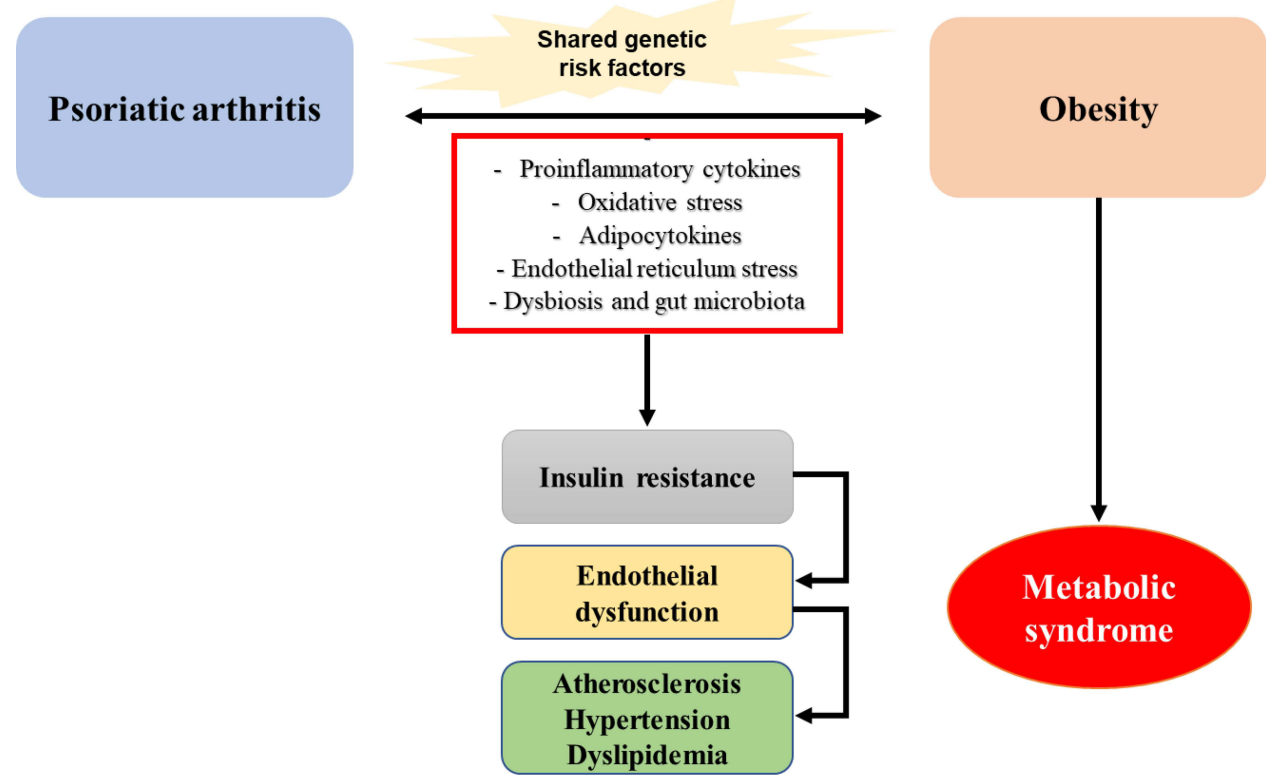

Figure I The link between inflammation, psoriatic arthritis, and metabolic syndrome; Obesity is one of the important components of metabolic syndrome and is a wellknown risk factor for psoriatic arthritis. Both obesity and PsA share a complex relationship that is likely bidirectional. Increased pro-inflammatory cytokines, secretion of adipocytokines from adipose tissue, increased of oxidative and endoplasmic reticulum stress, and dysbiosis of intestinal microbiota all play a role in the development of MetS and increase the risk of cardiovascular disease in psoriatic patients.

Metabolic syndrome has been discovered to be much more prevalent among people with PsA, PsO, and other inflammatory illnesses than in the overall population. ${ }^{32-34}$ Raychaudhuri et al explored in their study, which included 105 patients with PsA that 58.1\% of them diagnosed with MetS based on the AHA/NHLBI guidelines, and 61 of them satisfied a minimum of three out of five criteria for the diagnosis of the condition. ${ }^{35}$

Previous research by Haroon et al detected MetS in 44\% of 283 PsA patients. They also discovered that around half of those diagnosed with MetS showed $\geq 4$ MetS risk factors, with HTN (74\%), increased circumference of the waist (56\%), and hypertriglyceridemia (43.5\%) appearing more frequently. Moreover, the study found that MetS was correlated with increased odds in patients with severe underlying disease (OR 4.47, $\mathrm{p}=0.001){ }^{7}$

An increasing amount of clinical research has established that PsO is frequently linked with MetS. Patients with more extensive $\mathrm{PsO}$ were shown to have an increased chance to have MetS than those with mild disease. ${ }^{36-38}$ A large cohort study from Toronto investigated the MetS prevalence in PsA and compared them to PsO patients who did not have arthritis, finding a higher but not statistically significant prevalence of MetS in the former. ${ }^{39}$ Evidence from the literature and recent cross-sectional research indicates that those with PsA with apparent PsO had a higher frequency of MetS compared to PsA patients who did not have PsO $(40.48 \%$ vs $13.16 \%$, p $=0.006$, respectively). ${ }^{40}$

The association between MetS and chronic inflammatory arthritis has also been studied. According to research and growing evidence, higher CV risk is not only attributable to an increase in conventional risk factors; it is also associated with factors involving disease and its therapy, such as chronically high pro-inflammatory cytokines and prolonged glucocorticoid therapy. ${ }^{41,42}$

A large previous study on the MetS frequency incorporated 930 individuals diagnosed with chronic inflammatory arthritis, with PsA having the highest prevalence (38\%) followed by RA (20\%) and ankylosing spondylitis (11\%). Additionally, no significant correlation was discovered between MetS prevalence and disease longevity in the three inflammatory disorders. ${ }^{43}$ The frequency of MetS was also increasing in PsA patients than in the RA group in a large corona registry study ( $27 \%$ vs $19 \%$; $=0.02$, respectively), even after demographic variables and BMI adjustments. Moreover, among the components of MetS, diabetes, hypertriglyceridemia, and high BMI were more frequent in PsA patients. $^{33}$ 


\section{Psoriatic Arthritis and Obesity}

Obesity is a critical element of MetS that can ultimately cause other conditions to develop, including IR, HTN, and increased lipids. It was described as a chronic slow inflammatory state connected to elevated pro-inflammatory cytokine production that follows a common pathway with psoriatic diseases and has a detrimental effect on disease prognosis. ${ }^{44}$

The connection between adiposity and PsA is difficult to comprehend. For instance, PsA might increase the risk of obesity by decreasing physical activity levels due to the functional and psychological restrictions of the disease. In contrast, obesity may have occurred before PsA developed, emphasizing that it may be a risk factor for the disease. ${ }^{45}$

Previous serial case studies indicated an increased likelihood of developing PsA in individuals with PsO whose BMI was higher when they were young adults, regardless of other risk factors or current BMI. Hence, patients who had high BMI at the age of 18 are threefold more likely to acquire PsA during PsO course than those with an average weight at 18. However, the study did have several significant limitations, including the way PsA cases were defined, challenges in identifying a temporal connection between the examined factors, and insufficient verified data regarding how accurately patients could remember their BMI at age $18 .{ }^{46}$

$\mathrm{Li}$ et al conducted a 14-year prospective study that discovered a significant link between BMI and an increased incidence of PsA. Furthermore, they found an ascending positive relationship between weight changes started at the age of 18 and PsA risk. They also discovered an analogous relationship in individuals who acquired PsO during a study follow-up. $^{45}$

Additionally, a large population study showed a link between obesity and an increased chance of acquiring PsA in both $\mathrm{PsO}$ patients and the general population, regardless of other characteristics such as gender or age. ${ }^{47}$

\section{Insulin Resistance and Psoriatic Arthritis}

Insulin resistance is a significant cardiometabolic risk for CVS disease, which causes considerable morbidity and death in individuals with inflammatory arthritis. It is uncertain whether IR is a disease-specific feature or linked to high disease activity phases. Inflammatory cytokines, including TNF, IL-6, and IL1, which have frequent involvement in PSA pathophysiology, have been linked to IR in the liver and adipose tissues, impaired insulin action in human skeletal muscle, and an increased risk of DM. ${ }^{48,49}$

An observational cohort study found a significant prevalence of IR (16\%) among patients with PsA. Additionally, multiple regression analysis was conducted, the results of which indicated a significant relationship between IR and increased severity of underlying PsA disease, elevated BMI, and late-developing PsO. ${ }^{50}$ Furthermore, Eder et al discovered that diabetes mellitus was $43 \%$ more common in the PsA group than the total population. Notably, the severity of underlying psoriatic disease was identified as an important factor for developing diabetes across 11,006 overall person-years of follow-up. ${ }^{51}$

Additionally, Diabetes was shown to be more frequent in PsA patients than in sex- and age-matched persons without the disease (72\%, HR 1.72) in a large population-based cohort study. Interestingly, when controlling for BMI, alcohol consumption, smoking, initial glucocorticoid utilization, and co-morbidities, the relationship was considerably decreased but remained significant (33\%, HR 1.33) in PsA patients. ${ }^{52}$ As a result, the presence of inflammatory mediators cannot be entirely blamed for the development of diabetes; nevertheless, other variables, such as overweight and lifestyle habits, may also play an important role.

Furthermore, after controlling for atherosclerosis risk factors in individuals with PsA vs RA or ankylosing spondylitis, Mok et al discovered that PsA patients had more significant impairment in glucose tolerance (OR 2.58, P.001) compared to RA or ankylosing spondylitis patients. ${ }^{43}$

\section{Hypertension and Psoriatic Arthritis}

Hypertension is another essential component of MetS that is linked with an elevated risk of developing CVS disease. Studies indicated that HTN prevalence among PsA patients ranges from $25 \%$ to $49 \%{ }^{43,53,54}$ Furthermore, a large population study reported an increase in the frequency of HTN among those with PsA than in the general population (45.6\% vs $35.8 \%, \mathrm{P}<0.0001$ respectively). ${ }^{55}$ Moreover, individuals with PsA had a higher frequency of HTN than 
patients having only PsO (29\% vs $18 \%$, OR 1.7$).{ }^{56}$ Similarly, a study of a large cohort from the University of Toronto found also an increase in the HTN frequency among those with PsA in comparison to PsO patients with no arthritis (adjusted OR 2.17), even after controlling for known CVS traditional factors, medication history, as well as PsO duration and severity. ${ }^{32}$

In a large meta-analysis, the odds ratio of HTN was higher in individuals whose PsO was severe (OR 1.13) than those whose PsO was milder (OR1.09). Therefore, this finding emphasizes the significant contribution of inflammationpromoting arterial stiffness, which is linked to elevated hazard of $\mathrm{CV}$ events. ${ }^{57}$

Hypertension prevalence studies in patients diagnosed with different kinds of arthritis revealed a greater prevalence and incidence of HTN in PsA. A higher frequency (19.9\% vs 18.6\%) and incidence was reported in patients who had PsA than those with RA. Moreover, there was also an increased incidence of HTN among PsA patients in comparison to controls (HR 1.37) than among individuals with RA compared to controls (HR 1.16). ${ }^{11}$

\section{Dyslipidemia and Psoriatic Arthritis}

Dyslipidemia is well known as an independent cardiometabolic risk for CV disease. ${ }^{58}$ The relationship between dyslipidemia and PsA has been controversial and inconsistent in previous studies. One explanation for this might be that various definitions of dyslipidemia are used throughout the research, making it impossible to compare data concerning the relative prevalence. Furthermore, it has yet to be confirmed if dyslipidemia considered as a distinct cardiometabolic risk factor for CVD in PsA beyond obesity.

According to the findings of a large PsA cohort study conducted in the Middle East, participants with PsA had a greater prevalence of hyperlipidemia than the total population (OR 1.5, P.0001) ${ }^{55}$ In addition, Kimhi et al discovered a rise in the prevalence of hypertriglyceridemia, hypercholesterolemia, and low-density lipoprotein (LDL) among PsA patients in their study $\left(\mathrm{p}<0.005, \mathrm{p}<0.0001\right.$, and $\mathrm{p}<0.0001$, respectively).$^{53}$ Based on data from a previous study on PsA, hypercholesterolemia and hypertriglyceridemia were linked with subclinical atherosclerosis. ${ }^{42}$

In large HUNT research, hypertriglyceridemia was more frequent in PsA groups when compared to controls. However, it was not statistically relevant after controlling for BMI. Other lipid values were comparable in both groups. ${ }^{59}$ In contrast, another study indicated that PsA patients had reduced total and LDL cholesterol levels but increased highdensity lipoprotein (HDL) cholesterol, apolipoprotein A1 (Apo A1), and Apo B levels in PsA patients than in controls. Moreover, after controlling for BMI and hsCRP, the Apo B/Apo A1 ratio was considerably more elevated among the PsA groups than in the control groups. However, triglyceride levels were comparable across the two groups. ${ }^{54}$

Apo A1 is regarded to be a significant indicator of increased CV events with possible benefits above HDL cholesterol since it is responsible for transferring and functioning as the principal protein inhibiting atherogenesis in HDL particles. ${ }^{60}$

Apo B also carries all particles of lipoproteins that are potentially atherogenic. Although there is no consensus on the Apo B/Apo A1 ratio real use in previous studies, the cholesterol balance calculated based on the ratio has been demonstrated to be a superior measure compared with total lipids and lipoproteins levels. ${ }^{61}$ There is not yet a comprehensive understanding of the mechanisms behind these alterations in patients' lipid levels with inflammatory diseases in the literature.

Additionally, multiple factors influence serum lipid levels, such as the patient's diet and their usage of biologics or DMARDs. Apo B and triglyceride were elevated in those with PsA after commencing therapy with TNF inhibitor (TNFi), indicating a possible link between inflammation intensity and lipid abnormalities. ${ }^{62}$ Thus, because disease activity and anti-inflammatory medications can cause changes in lipid components, it is recommended that lipid profiling should ideally be performed when a patient is in stable condition or has entered remission. ${ }^{63}$

\section{Metabolic Syndrome and Cardiovascular Risk in Psoriatic Arthritis}

Metabolic syndrome raises the hazard of CV disease and atherosclerosis in PsA patients. ${ }^{64-67}$ A persistent underlying inflammatory state and pathogenetic pathways are shared by MetS, atherosclerosis, and inflammatory arthropathies. ${ }^{68,69}$ A persistent low-grade inflammatory condition with imbalanced immuno-inflammatory pathways and fatty acid metabolism in the artery wall promotes endothelial dysfunction. ${ }^{70}$ 
An increase in the carotid artery intima-media thickness(c-IMT), a predictor of pre-atherosclerosis with greater sensitivity, has been seen in PsA. More crucially, a higher risk of inflammation has been linked to the production of atherosclerotic plaques in individuals with PsA over time..$^{71-73}$

Those with PsA disease had a greater likelihood of atherosclerosis, endothelial dysfunction, and c-IMT, both with or without MetS components. ${ }^{42,53}$ Atherosclerosis is also linked to the severity of both PsA and MetS components, including obesity, diabetes, and hypertension. ${ }^{53}$

In addition, those with PsA had a greater frequency of MetS and significantly higher c-IMT levels than people with PsO. Furthermore, when PsA and PsO patients with or without MetS were compared, it was shown that PsA individuals with the coexistence of MetS had the highest c-IMT levels. ${ }^{74}$

In PsA patients, it has been established that the existence of carotid atherosclerosis has a clear association with CV events, which is related to the duration of PsA as well as the inflammatory condition, and it materializes regardless of the existence of conventional CV risk factors. ${ }^{54,64}$ Thus, chronic inflammation is crucial for the advancement of atherosclerosis in PsA, which works in an independent and/or synergistic manner with traditional risk factors. Furthermore, Eder et al demonstrated that the c-IMT and carotid plaque area were better than the Framingham risk score in predicting an elevated hazard of acquiring CV events in a population of PsA, which adds credence to the hypothesis that CV morbidity is not purely mediated by standard CVD risk factors in such patients. ${ }^{75}$

\section{Effect of Therapy on Metabolic Syndrome Components in Psoriatic Arthritis}

Metabolic syndrome and PsA may have a shared predilection for low-grade inflammation; thus, it is crucial to find a suitable therapy that effectively decreases both clinical and subclinical chronic inflammation.

Data from previous studies support the role played by Methotrexate (MTX) in lowering inflammation in patients with chronic inflammatory conditions and consequently reducing CV risk. However, most of the data were derived from rheumatoid arthritis patients. ${ }^{76,77}$

An observational study that included patients diagnosed with inflammatory arthritis and confirmed to have endothelial dysfunction indicated that all patients experienced a marked improvement in endothelium function following six months of therapy with MTX monotherapy, MTX combined with TNFi, or only TNFi. Nevertheless, after six months of therapy, endothelial function improvement was more sustained and significant in the MTX- treated patient compared to those treated with combination therapy. ${ }^{78}$

A 12-week pilot study explored the safety of MTX in terms of glucose metabolism in PsA and MetS patients and reported no difference in glycated hemoglobin levels before and after therapy. Thus, MTX usage in this patient group is safe, with no hyperglycemic consequences. In fact, MTX seems to have a beneficial effect on glucose metabolism because commencing MTX therapy reduced glycated hemoglobin levels compared to the use of metformin. ${ }^{79}$

There is significant debate surrounding the efficacy of TNFi in MetS patients. Numerous studies have demonstrated that TNFi is less effective in obese people. ${ }^{80}$ In a large meta-analysis, a relationship was established between being an obese and inadequate response to treatment among PsA patients. ${ }^{80}$ Additionally, Obesity was linked to reduced chances of attenuating lower disease activity in PsA, highlighting the critical need of patients with PsA decreasing weight. ${ }^{81}$ In contrast, data from another study discovered that the coexistence of MetS did not influence the anti-inflammatory role of TNFi or the risk that minimal disease activity would be attained. ${ }^{82}$

In a cohort study in which the follow-up period was 24 months, Costa et al found that patients who received etanercept (ETN) and adalimumab treatment exhibited considerable improvements in metabolic syndrome components (circumference of the waist, triglycerides, HDL-C, and glucose) in comparison to MTX group. ${ }^{83}$ Furthermore, some few studies have revealed that patients managed with TNFi had a decreased chance of getting diabetes than those managed with disease-modifying antirheumatic medication (except hydroxychloroquine) ${ }^{84}$

The influence of TNFi on the cholesterol level in individuals with the underlying chronic rheumatic disease remains unclear; a comprehensive meta-analysis identified that the use of TNFi increased total cholesterol by $10 \%$ and HDL-C by $7 \%$ over six months. Moreover, LDL-C and ApoB levels have been shown to increase in small studies. ${ }^{85}$ 
Agca et al evaluated 118 PsA patients who had received ETN treatment for a period exceeding five years. They found that LDL-C, HDL-C, and triglyceride levels were all increased. Additionally, the ratio for ApoB to ApoAI was reduced, a finding that may have clinical implications given that an elevated ratio is linked to an increased likelihood of CVD events. $^{86}$

Numerous large population-based studies have demonstrated that TNFi reduces the frequency of CV events in patient with psoriatic diseases. Armstrong et al found that PsO patients who used TNFi had a lower risk of MI than those who solely used topical therapy. ${ }^{87}$ In another study from Denmark, TNFi and MTX were linked with lower CV risk relative to topical medicines and phototherapy in $\mathrm{PsO}$ patients. ${ }^{88}$

In accordance with these findings, $\mathrm{Wu}$ and Poon found that patients with Psoriatic diseases who used TNFi had a reduced risk of new $\mathrm{CV}$ events than those who used other systemic medications or phototherapy. ${ }^{89}$

So, TNFi appears to have CV safety and maybe benefit, and it is favored over other treatments in patients at elevated $\mathrm{CV}$ risk, even though relatively little is known.

There is not enough evidence to indicate whether inhibiting IL-17 or IL12/23 improves metabolic syndrome in PsA patients. However, it has been demonstrated that being obese promotes the growth of T cells that produce IL-17 in fat and peripheral tissues. Furthermore, in patients with MetS, the IL-17R expression levels in the skeletal muscles and liver were linked to insulin resistance. ${ }^{56}$ Therefore, according to a large multi-center study, PsO patients who are obese have a lower response to secukinumab. ${ }^{90}$ However, another study found that obese PsA individuals may respond better to secukinumab than non-obese patients. ${ }^{91}$

Concern has been raised about the anti-IL-12/23 class of medications and the incidence of CV disease in PsA. However, a large, randomized trial on people with PsO found no association between ustekinumab usage and the risk of CV disease. ${ }^{92}$ More evidence on the CV safety of anti-IL-12/23 medications in psoriatic individuals is needed.

For therapy in PsA patients to be optimized, it is necessary to treat the skin and joint condition and identify and effectively manage co-morbidities. ${ }^{63}$ Much additional research must be done to fully grasp the complicated connection between PsA and MetS. The specific PsA variables linked to MetS must be identified, and it must be determined whether anti-inflammatory treatment approaches have any beneficial effect on MetS. Additionally, despite the existence of various DMARDs for the treatment of PsA, other factors, including the presence of co-morbidities, particularly MetS and CVS disease, must be taken into account when choosing suitable drugs for PsA management. ${ }^{93,94}$ The European Alliance of Associations for Rheumatology strongly encourages the adoption of lifestyle changes to mitigate CV risk factors. ${ }^{63}$ However, more investigation is needed to determine whether these strategies can augment or alter the effect of therapy on patients with MetS burden.

\section{Conclusion}

Psoriatic arthritis can present with various extra-articular symptoms that can manifest in isolation or together and that may take variable courses. Metabolic syndrome is intricately linked to systemic inflammation and has multiple inflammatory pathways in common with Psoriatic disease. The link between several components of MetS and psoriatic arthritis has been established, addressing the needs of screening, evaluating, and closely monitoring for co-morbidities. Therefore, rheumatologists should collaborate with other experts to detect MetS and its components as early as possible. In PsA patients with co-morbidities, the most appropriate therapy must be chosen for managing disease activity. Lifestyle changes, including smoking cessation and weight loss, are essential to decreasing CV events in PsA patients.

\section{Disclosure}

The author reports no conflicts of interest in this work.

\section{References}

1. Gudu T, Gossec L. Quality of life in psoriatic arthritis. Expert Rev Clin Immunol. 2018;14(5):405-417. doi:10.1080/1744666X.2018.1468252

2. Lee S, Mendelsohn A, Sarnes E. The burden of psoriatic arthritis: a literature review from a global health systems perspective. $P T$. 2010;35 (12):680-689.

3. Rendon A, Schäkel K. Psoriasis pathogenesis and treatment. Int J Mol Sci. 2019;20(6):1-28. doi:10.3390/ijms20061475 
4. Menter A, Griffiths CEM, Tebbey PW, Horn EJ, Sterry W. Exploring the association between cardiovascular and other disease-related risk factors in the psoriasis population: the need for increased understanding across the medical community. J Eur Acad Dermatology Venereol. 2010;24 (12):1371-1377. doi:10.1111/j.1468-3083.2010.03656.x

5. Jamnitski A, Visman IM, Peters MJL, Boers M, Dijkmans BAC, Nurmohamed MT. Prevalence of cardiovascular diseases in psoriatic arthritis resembles that of rheumatoid arthritis. Ann Rheum Dis. 2011;70(5):875LP-876. doi:10.1136/ard.2010.136499

6. Crăciun L, Crăciun P, Buicu F. Prevalence of metabolic syndrome in psoriatic arthritis and rheumatoid arthritis. Acta Medica Marisiensis. 2014;60 (5):196-199. doi:10.2478/amma-2014-0041

7. Haroon M, Gallagher P, FitzGerald O. SAT0293 high prevalence of metabolic syndrome in psoriatic arthritis patients is associated with severity of underlying psoriatic disease. Ann Rheum Dis. 2013;72(Supp13):A682-A683. doi:10.1136/annrheumdis-2013-eular.2018

8. Tobin AM, Veale DJ, FitzGerald O, et al. Cardiovascular disease and risk factors in patients with psoriasis and psoriatic arthritis. $J$ Rheumatol. 2010;37(7):1386-1394. doi:10.3899/jrheum.090822

9. Ogdie A, Schwartzman S, Husni ME. Recognizing and managing comorbidities in psoriatic arthritis. Curr Opin Rheumatol. 2015;27(2):118-126. doi:10.1097/BOR.0000000000000152

10. McHugh NJ, Balachrishnan C, Jones SM. Progression of peripheral joint disease in psoriatic arthritis: a 5-yr prospective study. Rheumatology. 2003;42(6):778-783. doi:10.1093/rheumatology/keg217

11. Radner H, Lesperance T, Accortt NA, Solomon DH. Incidence and prevalence of cardiovascular risk factors among patients with rheumatoid arthritis, psoriasis, or psoriatic arthritis. Arthritis Care Res. 2017;69(10):1510-1518. doi:10.1002/acr.23171

12. Alberti KGMM, Zimmet P, Shaw J. The metabolic syndrome - A new worldwide definition. Lancet. 2005;366(9491):1059-1062. doi:10.1016/ S0140-6736(05)67402-8

13. Grundy SM, Cleeman JI, Daniels SR, et al. Diagnosis and management of the metabolic syndrome: an American Heart Association/National Heart, Lung, and Blood Institute scientific statement. Circulation. 2005;112(17):2735-2752. doi:10.1161/CIRCULATIONAHA.105.169404

14. Expert Panel on Detection, Evaluation. Executive summary of the third report of the National Cholesterol Education Program (NCEP) expert panel on detection, evaluation, and treatment of high blood cholesterol in adults (adult treatment panel III). JAMA. 2001;285(19):2486-2497. doi:10.1001/jama.285.19.2486

15. Meigs JB, Rutter MK, Sullivan LM, Fox CS, D’Agostino RB, Wilson PWF. Impact of insulin resistance on risk of type 2 diabetes and cardiovascular. Diabetes Care. 2007;30(5):1219-1225. doi:10.2337/dc06-2484.J.B.M

16. Kruithof E, Baeten D, De Rycke L, et al. Synovial histopathology of psoriatic arthritis, both oligo- and polyarticular, resembles spondyloarthropathy more than it does rheumatoid arthritis. Arthritis Res Ther. 2005;7(3):R569. doi:10.1186/ar1698

17. Goodman WA, Levine AD, Massari JV, Sugiyama H, McCormick TS, Cooper KD. IL-6 signaling in psoriasis prevents immune suppression by regulatory T cells. J Immunol. 2009;183(5):3170-3176. doi:10.4049/jimmunol.0803721

18. Dao MC, Everard A, Aron-Wisnewsky J, et al. Akkermansia muciniphila and improved metabolic health during a dietary intervention in obesity: relationship with gut microbiome richness and ecology. Gut. 2016;65(3):426-436. doi:10.1136/gutjnl-2014-308778

19. Costello M-E, Robinson PC, Benham H, Brown MA. The intestinal microbiome in human disease and how it relates to arthritis and spondyloarthritis. Best Pract Res Clin Rheumatol. 2015;29(2):202-212. doi:10.1016/j.berh.2015.08.001

20. Jin M, Qian Z, Yin J, Xu W, Zhou X. The role of intestinal microbiota in cardiovascular disease. J Cell Mol Med. 2019;23(4):2343-2350. doi:10.1111/jcmm.14195

21. Rinninella E, Raoul P, Cintoni M, et al. What is the healthy gut microbiota composition? A changing ecosystem across age, environment, diet, and diseases. Microorganisms. 2019;7(1):14. doi:10.3390/microorganisms7010014

22. Qin J, Li Y, Cai Z, et al. A metagenome-wide association study of gut microbiota in type 2 diabetes. Nature. 2012;490(7418):55-60. doi:10.1038/ nature 11450

23. Ley RE, Backhed F, Turnbaugh P, Lozupone CA, Knight RD, Gordon JI. Obesity alters gut microbial ecology. Proc Natl Acad Sci. 2005;102 (31):11070-11075. doi:10.1073/pnas.0504978102

24. Cho CE, Taesuwan S, Malysheva OV, et al. Trimethylamine- N -oxide (TMAO) response to animal source foods varies among healthy young men and is influenced by their gut microbiota composition: a randomized controlled trial. Mol Nutr Food Res. 2017;61(1):1600324. doi:10.1002/ mnfr.201600324

25. Wang Z, Klipfell E, Bennett BJ, et al. Gut flora metabolism of phosphatidylcholine promotes cardiovascular disease. Nature. 2011;472(7341):5763. doi:10.1038/nature09922

26. Yan D, Issa N, Afifi L, Jeon C, Chang H-W, Liao W. The role of the skin and gut microbiome in psoriatic disease. Curr Dermatol Rep. 2017;6 (2):94-103. doi:10.1007/s13671-017-0178-5

27. Rodríguez-Cerdeira C, Cordeiro-Rodríguez M, Carnero-Gregorio M, et al. Biomarkers of inflammation in obesity-psoriatic patients. Mediators Inflamm. 2019;2019:1-14. doi:10.1155/2019/7353420

28. Trayhurn P, Wood IS. Adipokines: inflammation and the pleiotropic role of white adipose tissue. Br J Nutr. 2004;92(3):347-355. doi:10.1079/ bjn20041213

29. Hutcheson J. Adipokines influence the inflammatory balance in autoimmunity. Cytokine. 2015;75(2):272-279. doi:10.1016/j.cyto.2015.04.004

30. Chiricozzi A, Raimondo A, Lembo S, et al. Crosstalk between skin inflammation and adipose tissue-derived products: pathogenic evidence linking psoriasis to increased adiposity. Expert Rev Clin Immunol. 2016;12(12):1299-1308. doi:10.1080/1744666X.2016.1201423

31. Hong J, Maron DJ, Shirai T, Weyand CM. Accelerated atherosclerosis in patients with chronic inflammatory rheumatologic conditions. Int J Clin Rheumtol. 2015;10(5):365-381. doi:10.2217/ijr.15.33

32. Husted JA, Thavaneswaran A, Chandran V, et al. Cardiovascular and other comorbidities in patients with psoriatic arthritis: a comparison with patients with psoriasis. Arthritis Care Res. 2011;63(12):1729-1735. doi:10.1002/acr.20627

33. Labitigan M, Bahče-Altuntas A, Kremer JM, et al. Higher rates and clustering of abnormal lipids, obesity, and diabetes mellitus in psoriatic arthritis compared with rheumatoid arthritis. Arthritis Care Res. 2014;66(4):600-607. doi:10.1002/acr.22185

34. Loganathan A, Kamalaraj N, El-Haddad C, Pile K. Systematic review and meta-analysis on prevalence of metabolic syndrome in psoriatic arthritis, rheumatoid arthritis and psoriasis. Int J Rheum Dis. 2021;24(9):1112-1120. doi:10.1111/1756-185X.14147

35. Raychaudhuri SK, Chatterjee S, Nguyen C, Kaur M, Jialal I, Raychaudhuri SP. Increased prevalence of the metabolic syndrome in patients with psoriatic arthritis. Metab Syndr Relat Disord. 2010;8(4):331-334. doi:10.1089/met.2009.0124 
36. Rodríguez-Zúñiga MJM, García-Perdomo HA. Systematic review and meta-analysis of the association between psoriasis and metabolic syndrome. J Am Acad Dermatol. 2017;77(4):657-666.e8. doi:10.1016/j.jaad.2017.04.1133

37. Armstrong AW, Harskamp CT, Armstrong EJ. The association between psoriasis and obesity: a systematic review and meta-analysis of observational studies. Nutr Diabetes. 2012;2(12):e54. doi:10.1038/nutd.2012.26

38. Caroppo F, Galderisi A, Moretti C, Ventura L, Belloni Fortina A. Prevalence of psoriasis in a cohort of children and adolescents with type 1 diabetes. J Eur Acad Dermatology Venereol. 2021;35(9):1-3. doi:10.1111/jdv.17318

39. Eder L, Jayakar J, Pollock R, et al. Serum adipokines in patients with psoriatic arthritis and psoriasis alone and their correlation with disease activity. Ann Rheum Dis. 2013;72(12):1956-1961. doi:10.1136/annrheumdis-2012-202325

40. Caso F, Del Puente A, Oliviero F, et al. Metabolic syndrome in psoriatic arthritis: the interplay with cutaneous involvement. Evidences from literature and a recent cross-sectional study. Clin Rheumatol. 2018;37(3):579-586. doi:10.1007/s10067-017-3975-0

41. Del Rincón I, Freeman GL, Haas RW, O’Leary DH, Escalante A. Relative contribution of cardiovascular risk factors and rheumatoid arthritis clinical manifestations to atherosclerosis. Arthritis Rheum. 2005;52(11):3413-3423. doi:10.1002/art.21397

42. Gonzalez-juanatey C, Llorca J, Amigo-Diaz E, Dierssen T, Martin J, Gonzalez-Gay MA. High prevalence of subclinical atherosclerosis in psoriatic arthritis patients without clinically evident cardiovascular disease or classic atherosclerosis risk factors. Arthritis Rheum. 2007;57(6):1074-1080. doi:10.1002/art.22884

43. Mok CC, Ko GTC, Ho LY, Yu KL, Chan PT, To CH. Prevalence of atherosclerotic risk factors and the metabolic syndrome in patients with chronic inflammatory arthritis. Arthritis Care Res. 2011;63(2):195-202. doi:10.1002/acr.20363

44. Ellulu MS, Patimah I, Khaza'ai H, Rahmat A, Abed Y. Obesity and inflammation: the linking mechanism and the complications. Arch Med Sci. 2017;4:851-863. doi:10.5114/aoms.2016.58928

45. Li W, Han J, Qureshi AA. Obesity and risk of incident psoriatic arthritis in US women. Ann Rheum Dis. 2012;71(8):1267-1272. doi:10.1136/ annrheumdis-2011-201273

46. Soltani-Arabshahi R, Wong B, Feng BJ, Goldgar DE, Callis Duffin K, Krueger GG. Obesity in early adulthood as a risk factor for psoriatic arthritis. Arch Dermatol. 2010;146(7):721-726. doi:10.1001/archdermatol.2010.141

47. Jon Love T, Zhu Y, Zhang Y, et al. Obesity and the risk of psoriatic arthritis: a population-based study. Ann Rheum Dis. 2012;71(8):1273-1277. doi:10.1136/annrheumdis-2012-201299

48. Ormazabal V, Nair S, Elfeky O, Aguayo C, Salomon C, Zuñiga FA. Association between insulin resistance and the development of cardiovascular disease. Cardiovasc Diabetol. 2018;17(1):1-14. doi:10.1186/s12933-018-0762-4

49. Rotter V, Nagaev I, Smith U. Interleukin-6 (IL-6) induces insulin resistance in 3T3-L1 adipocytes and is, like IL-8 and tumor necrosis factor- $\alpha$, overexpressed in human fat cells from insulin-resistant subjects. J Biol Chem. 2003;278(46):45777-45784. doi:10.1074/jbc.M301977200

50. Haroon M, Gallagher P, Heffernan E, FitzGerald O. High prevalence of metabolic syndrome and of insulin resistance in psoriatic arthritis is associated with the severity of underlying disease. $J$ Rheumatol. 2014;41(7):1357-1365. doi:10.3899/jrheum.140021

51. Eder L, Chandran V, Cook R, Gladman DD. The risk of developing diabetes mellitus in patients with psoriatic arthritis: a cohort study. $J$ Rheumatol. 2017;44(3):286-291. doi:10.3899/jrheum.160861

52. Dubreuil M, Rho YH, Man A, et al. Diabetes incidence in psoriatic arthritis, psoriasis and rheumatoid arthritis: a UK population-based cohort study. Rheumatology. 2014;53(2):346-352. doi:10.1093/rheumatology/ket343

53. Kimhi O, Caspi D, Bornstein NM, et al. Prevalence and risk factors of atherosclerosis in patients with psoriatic arthritis. Semin Arthritis Rheum. 2007;36(4):203-209. doi:10.1016/j.semarthrit.2006.09.001

54. Tam LS, Tomlinson B, Chu TTW, et al. Cardiovascular risk profile of patients with psoriatic arthritis compared to controls - The role of inflammation. Rheumatology. 2008;47(5):718-723. doi:10.1093/rheumatology/ken090

55. Kibari A, Cohen AD, Gazitt T, et al. Cardiac and cardiovascular morbidities in patients with psoriatic arthritis: a population-based case control study. Clin Rheumatol. 2019;38(8):2069-2075. doi:10.1007/s10067-019-04528-y

56. Queiro R, Lorenzo A, Tejón P, et al. Obesity in psoriatic arthritis. Med. 2019;98(28):e16400. doi:10.1097/MD.0000000000016400

57. Duan X, Liu J, Mu Y, et al. A systematic review and meta-analysis of the association between psoriasis and hypertension with adjustment for covariates. Med. 2020;99(9). doi:10.1097/MD.0000000000019303

58. Rohold A, Haastrup B, Larsen S, Hansen AB, Larsen ML, Haghfelt T. Dyslipidemia and coronary artery disease. Cardiology. 1996;87(6):497-501. doi:10.1159/000177145

59. Gulati AM, Semb AG, Rollefstad S, et al. On the HUNT for cardiovascular risk factors and disease in patients with psoriatic arthritis: populationbased data from the Nord-Trøndelag Health Study. Ann Rheum Dis. 2016;75(5):819-824. doi:10.1136/annrheumdis-2014-206824

60. Lu M, Lu Q, Zhang Y, Tian G. ApoB/apoA1 is an effective predictor of coronary heart disease risk in overweight and obesity. $J$ Biomed Res. 2011;25(4):266-273. doi:10.1016/S1674-8301(11)60036-5

61. Walldius G, Jungner I. The apoB/apoA-I ratio: a strong, new risk factor for cardiovascular disease and a target for lipid-lowering therapy - a review of the evidence. J Intern Med. 2006;259(5):493-519. doi:10.1111/j.1365-2796.2006.01643.x

62. Sattar N, Crompton P, Cherry L, Kane D, Lowe G, McInnes IB. Effects of tumor necrosis factor blockade on cardiovascular risk factors in psoriatic arthritis: a double-blind, placebo-controlled study. Arthritis Rheum. 2007;56(3):831-839. doi:10.1002/art.22447

63. Agca R, Heslinga SC, Rollefstad S, et al. EULAR recommendations for cardiovascular disease risk management in patients with rheumatoid arthritis and other forms of inflammatory joint disorders: 2015/2016 update. Ann Rheum Dis. 2016;76(1):17-28. doi:10.1136/annrheumdis-2016209775

64. Eder L, Thavaneswaran A, Chandran V, Cook R, Gladman DD. Increased burden of inflammation over time is associated with the extent of atherosclerotic plaques in patients with psoriatic arthritis. Ann Rheum Dis. 2015;74(10):1830-1835. doi:10.1136/annrheumdis-2014-205267

65. Peluso R, Caso F, Tasso M, et al. Cardiovascular risk markers and major adverse cardiovascular events in psoriatic arthritis patients. Rev Recent Clin Trials. 2018;13(3):199-209. doi:10.2174/1574887113666180314105511

66. Gladman DD, Ang M, Su L, Tom BDM, Schentag CT, Farewell VT. Cardiovascular morbidity in psoriatic arthritis. Ann Rheum Dis. 2009;68 (7):1131-1135. doi:10.1136/ard.2008.094839

67. Eder L, Harvey P. Cardiovascular morbidity in psoriatic arthritis: what is the effect of inflammation? J Rheumatol. 2017;44(9):1295-1297. doi:10.3899/jrheum.170534 
68. Eder L, Gladman DD. Atherosclerosis in psoriatic disease: latest evidence and clinical implications. Ther Adv Musculoskelet Dis. 2015;7(5):187195. doi:10.1177/1759720X15591801

69. Ruscitti P, Cipriani P, Liakouli V, et al. Subclinical and clinical atherosclerosis in rheumatoid arthritis: results from the 3-year, multicentre, prospective, observational GIRRCS (Gruppo Italiano di Ricerca in Reumatologia Clinica e Sperimentale) study. Arthritis Res Ther. 2019;21(1):110. doi:10.1186/s13075-019-1975-y

70. Ramji DP, Davies TS. Cytokines in atherosclerosis: key players in all stages of disease and promising therapeutic targets. Cytokine Growth Factor Rev. 2015;26(6):673-685. doi:10.1016/j.cytogfr.2015.04.003

71. Baldassarre D, Amato M, Bondioli A, Sirtori CR, Tremoli E. Carotid artery intima-media thickness measured by ultrasonography in normal clinical practice correlates well with atherosclerosis risk factors. Stroke. 2000;31(10):2426-2430. doi:10.1161/01.STR.31.10.2426

72. Shen J, Shang Q, Li EK, et al. Cumulative inflammatory burden is independently associated with increased arterial stiffness in patients with psoriatic arthritis: a prospective study. Arthritis Res Ther. 2015;17(1):1-9. doi:10.1186/s13075-015-0570-0

73. Costa L, Caso F, D'Elia L, et al. Psoriatic arthritis is associated with increased arterial stiffness in the absence of known cardiovascular risk factors: a case control study. Clin Rheumatol. 2012;31(4):711-715. doi:10.1007/s10067-011-1892-1

74. Lin YC, Dalal D, Churton S, et al. Relationship between metabolic syndrome and carotid intima-media thickness: cross-sectional comparison between psoriasis and psoriatic arthritis. Arthritis Care Res. 2014;66(1):97-103. doi:10.1002/acr.22144

75. Eder L, Zisman D, Barzilai M, et al. Subclinical atherosclerosis in psoriatic arthritis: a case-control study. J Rheumatol. $2008 ; 35(5): 877-882$.

76. Bălănescu AR, Bojincă VC, Bojincă M, Donisan T, Bălănescu SM. Cardiovascular effects of methotrexate in immune-mediated inflammatory diseases (Review). Exp Ther Med. 2019;17(2):1024-1029. doi:10.3892/etm.2018.6992

77. Reiss AB, Carsons SE, Anwar K, et al. Atheroprotective effects of methotrexate on reverse cholesterol transport proteins and foam cell transformation in human THP-1 monocyte/macrophages. Arthritis Rheum. 2008;58(12):3675-3683. doi:10.1002/art.24040

78. Deyab G, Hokstad I, Whist JE, et al. Methotrexate and anti-tumor necrosis factor treatment improves endothelial function in patients with inflammatory arthritis. Arthritis Res Ther. 2017;19(1):1-10. doi:10.1186/s13075-017-1439-1

79. Dehpouri T, Rokni GR, Narenjbon NA, et al. Evaluation of the glycemic effect of methotrexate in psoriatic arthritis patients with metabolic syndrome: a pilot study. Dermatol Rep. 2019;11(1):22-26. doi:10.4081/dr.2019.7965

80. Singh S, Facciorusso A, Singh AG, et al. Obesity and response to anti-tumor necrosis factor- $\alpha$ agents in patients with select immune-mediated inflammatory diseases: a systematic review and meta-analysis. PLoS One. 2018;13(5):1-26. doi:10.1371/journal.pone.0195123

81. Ogdie A, Palmer JL, Greenberg J, et al. Predictors of achieving remission among patients with psoriatic arthritis initiating a tumor necrosis factor inhibitor. J Rheumatol. 2019;46(5):475-482. doi:10.3899/jrheum.171034

82. Eder L, Thavaneswaran A, Chandran V, Cook RJ, Gladman DD. Obesity is associated with a lower probability of achieving sustained minimal disease activity state among patients with psoriatic arthritis. Ann Rheum Dis. 2015;74(5):813-817. doi:10.1136/annrheumdis-2013-204448

83. Iervolino S, Di Minno MND, Peluso R, et al. Predictors of early minimal disease activity in patients with psoriatic arthritis treated with tumor necrosis factor- $\alpha$ blockers. J Rheumatol. 2012;39(3):568-573. doi:10.3899/jrheum.110763

84. Costa L, Caso F, Atteno M, et al. Impact of 24-month treatment with etanercept, Adalimumab, or methotrexate on metabolic syndrome components in a cohort of 210 psoriatic arthritis patients. Clin Rheumatol. 2014;33(6):833-839. doi:10.1007/s10067-013-2369-1

85. Huizinga T, Nigrovic P, Ruderman E, Schulze-Koops H. Association between disease-modifying antirheumatic drugs and diabetes risk in patients with rheumatoid arthritis and psoriasis: commentary. Int $J$ Adv Rheumatol. 2011;9(4):143.

86. Agca R, Heslinga M, Kneepkens EL, Van Dongen C, Nurmohamed MT. The effects of 5-year etanercept therapy on cardiovascular risk factors in patients with psoriatic arthritis. J Rheumatol. 2017;44(9):1362-1368. doi:10.3899/jrheum.161418

87. Armstrong AW. Do TNF inhibitors reduce the risk of myocardial infarction in psoriasis patients? JAMA. 2013;309(19):2043. doi:10.1001/ jama.2013.4695

88. Ahlehoff O, Skov L, Gislason G, et al. Cardiovascular outcomes and systemic anti-inflammatory drugs in patients with severe psoriasis: 5-year follow-up of a Danish nationwide cohort. J Eur Acad Dermatology Venereol. 2015;29(6):1128-1134. doi:10.1111/jdv.12768

89. Wu JJ, Poon KY. Tumor necrosis factor inhibitor therapy and myocardial infarction risk in patients with psoriasis, psoriatic arthritis, or both. $J$ Drugs Dermatol. 2014;13(8):932-934.

90. Szepietowski J, Rich P, Loeffler J, et al. Secukinumab $300 \mathrm{mg}$ shows superior efficacy across subject body weight groups: pooled analysis of phase 3 ERASURE and FIXTURE trials. $J$ Am Acad Dermatol. 2015;72(5):AB248.

91. Notario J, Deza G, Vilarrasa E, et al. Treatment of patients with plaque psoriasis with secukinumab in a real-life setting: a 52-week, multicenter, retrospective study in Spain. J Dermatolog Treat. 2019;30(5):424-429. doi:10.1080/09546634.2018.1528000

92. de Brito M, Yiu ZZN. Cardiovascular safety of biologics targeting interleukin (IL)-12 and/or IL-23: what does the evidence say? Am $J$ Clin Dermatol. 2021;22(5):587-601. doi:10.1007/s40257-021-00612-9

93. Pantano I, Iacono D, Favalli EG, et al. Secukinumab efficacy in patients with PsA is not dependent on patients' body mass index. Ann Rheum Dis. 2020:5-6. doi:10.1136/annrheumdis-2020-217251

94. Kaushik SB, Lebwohl MG. Psoriasis: which therapy for which patient: focus on special populations and chronic infections. J Am Acad Dermatol. 2019;80(1):43-53. doi:10.1016/j.jaad.2018.06.056

Open Access Rheumatology: Research and Reviews

Dovepress

Publish your work in this journal

Open Access Rheumatology Research and Reviews is an international, peer-reviewed, open access journal publishing original research, reports, editorials, reviews and commentaries on all aspects of clinical and experimental rheumatology in the clinic and laboratory including the following topics: Pathology, pathophysiology of rheumatological diseases; Investigation, treatment and management of rheumatological diseases; Clinical trials and novel pharmacological approaches for the treatment of rheumatological disorders. The manuscript management system is completely online and includes a very quick and fair peer-review system, which is all easy to use. Visit http://www.dovepress.com/ testimonials.php to read real quotes from published authors.

Submit your manuscript here: https://www.dovepress.com/open-access-rheumatology-research-and-reviews-journal 